\title{
Matriciamento em saúde mental na atenção primária: Uma revisão integrativa da
}

\section{literatura}

\author{
Mental health enrollment in primary care: An integrative literature review \\ Apoyo de la matriz de salud mental en la atención primaria: Una revisión integradora de la
}

literatura

Recebido: 30/03/2021 | Revisado: 08/04/2021 | Aceito: 20/04/2021 | Publicado: 06/05/2021

\author{
Amanda Ouriques de Gouveia \\ ORCID: https://orcid.org/0000-0002-6874-8352 \\ Fundação Santa Casa de Misericórdia do Pará, Brasil \\ E-mail: mandinhaouriques@hotmail.com \\ Carmen Lúcia de Araújo Paes \\ ORCID: https://orcid.org/0000-0001-9365-2451 \\ Fundação Santa Casa de Misericórdia do Pará, Brasil \\ E-mail: carmenaraujopaes@gmail.com \\ Valeria Regina Cavalcante dos Santos \\ ORCID: https://orcid.org/0000-0002-1264-8125 \\ Fundação Santa Casa de Misericórdia do Pará, Brasil \\ E-mail: valregsantos@hotmail.com \\ Ilma Pastana Ferreira \\ ORCID: https://orcid.org/0000-0002-9152-3872 \\ Fundação Santa Casa de Misericórdia do Pará, Brasil \\ E-mail: ilma.m@oi.com.br.
}

\begin{abstract}
Resumo
O Matriciamento surge como um novo modelo de produzir saúde, fazendo com que duas ou mais equipes desenvolvam uma proposta de intervenção pedagógico-terapêutica, num método de construção compartilhada. O objetivo deste estudo é conhecer as evidências na literatura dos últimos 06 anos referentes ao matriciamento em saúde mental na atenção primária, bem como os seus benefícios e possíveis dificuldades de implantação. Trata-se de um estudo descritivo, de abordagem qualitativa e caráter exploratório, do tipo Revisão Integrativo da Literatura (RIL). As bases de dados utilizadas foram a BDENF, LILACS e SCIELO. Após a aplicação dos critérios de inclusão e exclusão, dos 37 resultados nas bases eletrônicas de dados, obteve-se uma amostra de 15 estudos. A partir da análise dos artigos, foram identificadas duas categorias, escolhidas por agrupamentos de aproximação de contextos e ideias: 1) benefícios e resultados do modelo matricial; 2) fatores que interferem na implantação do matriciamento. Verificou-se que a maior parte dos estudos encontrados relatavam as contribuições da implantação do modelo matricial em saúde mental. No entanto, a falta de capacitação técnica, os estigmas e preconceitos, bem como a centralização e burocratização dos serviços dificultam a adesão desta estratégia.
\end{abstract}

Palavras-chave: Atenção primária à saúde; Modelo matricial; Saúde mental.

\begin{abstract}
Matricization appears as a new model to produce health, causing two or more teams to develop a pedagogicaltherapeutic intervention proposal, in a shared construction method. The objective of this study is to know the evidences in the literature of the last 06 years regarding mental health enrollment in primary care, as well as its benefits and possible difficulties of implantation. This is a descriptive study, with a qualitative approach and an exploratory nature, of the Integrative Literature Review (RIL) type. The databases used were BDENF, LILACS and SCIELO. After applying the inclusion and exclusion criteria, of the 37 results in the electronic databases, a sample of 15 studies was obtained. From the analysis of the articles, two categories were identified, chosen by clusters of approximation of contexts and ideas: 1) benefits and results of the matrix model; 2) factors that interfere in the implementation of the matrix. It was found that most of the studies reported the contributions of the implementation of the matrix model in mental health. However, the lack of technical training, stigmata and prejudices, as well as the centralization and bureaucratization of services make it difficult to adhere to this strategy.
\end{abstract}

Keywords: Primary health care; Matrix model; Mental health.

\section{Resumen}

El Matrixing surge como un nuevo modelo de producción de salud, provocando que dos o más equipos desarrollen una propuesta de intervención pedagógico-terapéutica, en un método de construcción compartida. El objetivo de este 
estudio es conocer la evidencia en la literatura de los últimos 06 años sobre el soporte de la matriz de salud mental en la atención primaria, así como sus beneficios y posibles dificultades de implementación. Se trata de un estudio descriptivo, con enfoque cualitativo y carácter exploratorio, del tipo Revisión de Literatura Integrativa (RIL). Las bases de datos utilizadas fueron BDENF, LILACS y SCIELO. Tras aplicar los criterios de inclusión y exclusión, de los 37 resultados en las bases de datos electrónicas se obtuvo una muestra de 15 estudios. A partir del análisis de los artículos se identificaron dos categorías, elegidas por grupos de aproximación de contextos e ideas: 1) beneficios y resultados del modelo matricial; 2) factores que interfieren con la implementación del soporte matricial. Se encontró que la mayoría de los estudios encontrados reportaron los aportes de la implementación del modelo matricial en salud mental. Sin embargo, la falta de formación técnica, los estigmas y prejuicios, así como la centralización y burocratización de los servicios dificultan la adhesión a esta estrategia.

Palabras clave: Primeros auxilios; Modelo matricial; Salud mental.

\section{Introdução}

Segundo o Ministério da Saúde, os transtornos mentais caracterizam-se por alterações de pensamento, emoções, comportamento que geram angústia ou interfiram na vida da pessoa. Dados da Organização Mundial da Saúde, afirmam que as doenças psíquicas respondem por cerca de um quarto de todos os anos perdidos por incapacidade no mundo e prever que, neste ano, aumentem e torne-se a segunda causa. Nesse viés, estás doenças representavam em 2010 aproximadamente 7,4\% dos problemas de saúde mundial (Brasil, 2011; Opas, 2010).

Nesse contexto, é importante ressaltar que os indivíduos portadores de transtornos mentais sempre existiram, e na maioria dos casos, foram marginalizados ou tratados de forma desumana. Nos manicômios, por exemplo os doentes viviam confinados até a sua morte e muitas vezes eram submetidos a tratamentos com técnicas violentas. Contudo, com a reforma psiquiátrica ocorrida em 1987, adveio uma reformulação nas propostas terapêuticas para esta população, na qual foram abolidos os tratamentos anteriormente realizados e substituídos por procedimentos que possibilitaram o cuidado em liberdade e o resgate da cidadania (Ortega \& Wenceslau, 2016).

Entretanto, mesmo com a abolição dos hospitais psiquiátricos, atualmente três em cada quatro pessoas com doenças mentais não recebem tratamento adequado ou nem mesmo são diagnosticadas com este problema. Percebe-se, portanto, que os transtornos mentais são um problema de saúde pública preocupante, levando em consideração a alta prevalência em contraponto com a escassez de serviços. Desse modo, as avaliações mundiais afirmam a necessidade de ampliação dos sistemas de saúde, já que a taxa dos que necessitam e não são tratados é elevada, sobretudo nos países em desenvolvimento (Faro et al, 2020).

Nessa conjuntura, o Ministério da Saúde propôs para a resolutividade do tema em questão o apoio matricial, que é um processo de integração e suporte de profissionais especialistas em saúde mental na Atenção Primária em Saúde (APS). O matriciamento, a partir disso surge como um novo modelo de produzir saúde, fazendo com que duas ou mais equipes desenvolvam uma proposta de intervenção pedagógico-terapêutica, num método de construção compartilhada. Ele pode ser realizado por profissionais de diversas áreas especializadas (Opas, 2010; Ortega \& Wenceslau, 2016).

Destarte, as equipes da Atenção Básica em especial das Estratégias de Saúde da Família (ESF), devem incluir ações de saúde mental em suas agendas, desse modo, conseguir-se-ia contempla a saúde mental e física do paciente, melhorando o acesso aos serviços de saúde mental, promovendo os direitos humanos e reduzindo a lacuna entre a prevalência desses transtornos e o número de pessoas atendidas, além de diminuir as filas de espera para consultas, consequentemente, amenizando o sofrimento dos doentes e familiares (Macedo \& Abreu, 2017).

Todavia, para que esse modelo de saúde mental seja desenvolvido é necessário que as equipes das APS sejam capacitadas, desse modo, adquirindo aptidão de comunicação, de manejo de problemas psicossociais e de acompanhamento, além da capacidade de detectar problemas em seu território e propor formas de intervenção adequadas. Assim, levando a inserção da saúde mental como prioridade de saúde pública e a sua integração aos serviços de saúde em geral. Portanto, 
favorecendo um cuidado integral com o paciente, que é umas das premissas do Sistema Único de Saúde. (Gryschek \& Pinto, 2015)

Isto posto, o presente estudo objetiva analisar estudos ao apoio matricial em saúde mental na atenção primária segundo as produções cientificas dos últimos seis anos, bem verificar os seus benefícios e possíveis dificuldades de sua implantação.

\section{Metodologia}

Trata-se de um estudo descritivo, de abordagem qualitativa e caráter exploratório, do tipo Revisão Integrativo da Literatura (RIL). A RIL segundo Souza et al, (2010) é realizada em seis etapas: elaboração da pergunta norteadora, busca ou amostragem na literatura, coleta de dados, análise crítica dos estudos incluídos, discussão dos resultados e apresentação da revisão integrativa.

Mediante aos objetivos propostos buscou-se responder às seguintes questões norteadoras: Que fatores interferem na implantação da estratégia de matriciamento em saúde mental na atenção primária? Quais os benefícios da adesão este modelo de atenção à saúde?

A procura pelos artigos deu-se com a utilização dos descritores "matriciamento", "saúde mental", "Assistência à Saúde Mental", "Equipe Multiprofissional" e "Atenção Primária", relacionados entre si com o operador boleano and. Tais termos foram indexados nas seguintes bases de dados: Base de Dados de Enfermagem (BDENF), Literatura Latino-Americana da Caribe em Ciências da Saúde (LILACS), PubMed, Google Acadêmico e Scientific Electronic Library Online (Scielo).

A partir disso, fora empregado como critério de inclusão artigos com limite de até cinco anos, em português e espanhol, com delineamento descritivo e qualitativo de levantamento normativo ou de análise de temáticas voltadas para as áreas que abordassem o matriciamento em saúde mental na atenção primária, como critérios de exclusão aplicou-se artigos que não se enquadravam nos critérios de inclusão.

A coleta de dados ocorreu entre os meses de outubro e novembro de 2020 e transcorreu em três fases, a primeira ocorreu a leitura dos resumos dos artigos, dos quais 78 foram pré-selecionados e posteriormente lidos, destes permanecendo 15 artigos. Em seguida, realizou-se uma releitura exaustiva dos materiais selecionados e registro das partes mais relevantes, para que ocorresse melhor compreensão e se conseguisse englobar todas as vertentes por eles expostas. A terceira fase caracterizouse pela formulação do artigo, como demostrado no Esquema 1. 
Esquema 1: Organograma do processo de produção do artigo.

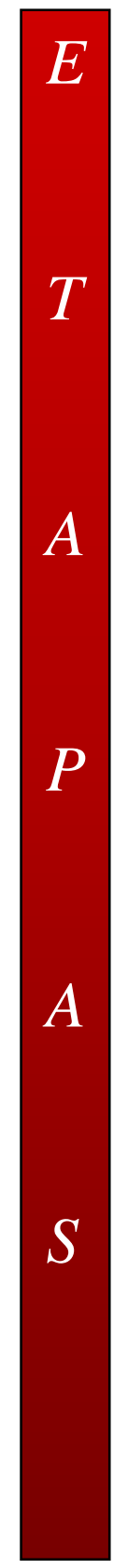

Busca pelos artigos

78 pré-selecionados

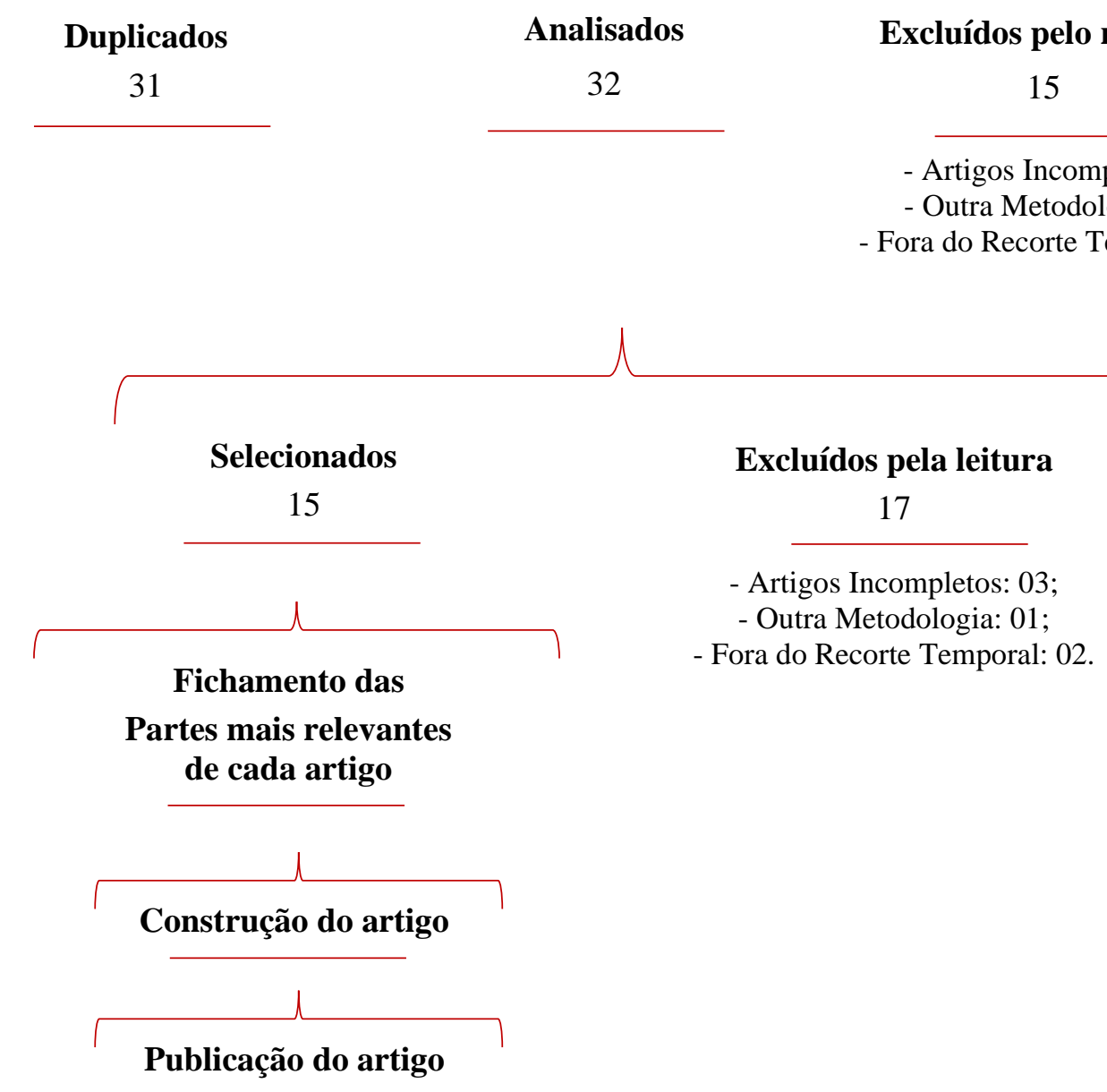

Fonte: Autores (2021).

\section{Resultados}

Após a aplicação dos critérios de inclusão e exclusão, dos 37 resultados nas bases eletrônicas de dados, obteve-se uma amostra de 15 estudos. Destes, verificou-se que 02 eram da base de dados BDENF, 06 da LILACS e 08 pertenciam à SCIELO.

Quanto a distribuição de publicações por ano, verificou-se, dentre os resultados, 02 no ano de 2016, 04 em 2017, 04 em 2018, 03 em 2019 e 02 no ano de 2020, conforme mostra o quadro 01. Embora o período deste estudo para as produções encontradas fosse referente ao período de 2015 a 2020, não foram encontradas referências no ano de 2015 nas bases eletrônicas supracitadas. Tal fato evidencia que quanto mais próximo dos dias atuais, maior é a produção referente ao modelo matricial em saúde mental na atenção primária, como demostrado no Quadro 1. 
Quadro 1: Distribuição de estudos por ano, base de dados, título, autores e descritores.

\begin{tabular}{|c|c|c|c|c|c|}
\hline $\mathbf{N}^{\mathbf{o}}$ & ANO & BASE & TÍTULO & AUTORES & DESCRITORES \\
\hline 01 & 2019 & BDENF & $\begin{array}{l}\text { Conhecimento de Gestores e } \\
\text { Profissionais da Rede de Atenção } \\
\text { Psicossocial sobre matriciamento em } \\
\text { saúde mental }\end{array}$ & $\begin{array}{l}\text { Vasconcelos, \& } \\
\text { Barbosa. }\end{array}$ & $\begin{array}{l}\text { Atenção primária à saúde. Saúde } \\
\text { mental. Gestão em Saúde. } \\
\text { Desinstitucionalização. }\end{array}$ \\
\hline 02 & 2020 & BDENF & $\begin{array}{l}\text { O cuidado aos portadores de } \\
\text { sofrimento mental na atenção } \\
\text { primária: uma prática interdisciplinar } \\
\text { e multiprofissional }\end{array}$ & Almeida et al. & $\begin{array}{l}\text { Assistência de Enfermagem. } \\
\text { Saúde Mental. Atenção primária. } \\
\text { Interdisciplinar. } \\
\text { Multiprofissional. }\end{array}$ \\
\hline 03 & 2016 & LILACS & $\begin{array}{l}\text { A prática do apoio matricial e os seus } \\
\text { efeitos na Atenção Primária à Saúde }\end{array}$ & Belotti \& Lavrador, & $\begin{array}{l}\text { Saúde Mental. Saúde da Família. } \\
\text { Atenção à Saúde. }\end{array}$ \\
\hline 04 & 2020 & LILACS & $\begin{array}{c}\text { Apoio matricial como Ferramenta da } \\
\text { Articulação entre Atenção Básica e } \\
\text { CAPS }\end{array}$ & Godoi & $\begin{array}{l}\text { Atenção primária à saúde. Saúde } \\
\text { Mental. Integração de sistemas. } \\
\text { Serviços de Saúde. }\end{array}$ \\
\hline 05 & 2017 & LILACS & $\begin{array}{l}\text { Implantação do matriciamento em } \\
\text { saúde mental na atençâo bàsica do } \\
\text { municìpio de Toritama-PE }\end{array}$ & Silva et al. & $\begin{array}{l}\text { Saúde Mental. Atenção Básica. } \\
\text { Educação } \\
\text { Matriciamento. }\end{array}$ \\
\hline 06 & 2018 & LILACS & $\begin{array}{l}\text { Matriciamento em saúde mental: } \\
\text { obstáculos, caminhos e resultados }\end{array}$ & Gonçalves \& Peres. & $\begin{array}{l}\text { Saúde mental. Apoio matricial. } \\
\text { Centro de Atenção Psicossocial. } \\
\text { Serviços de saúde pública. } \\
\text { Revisão de literatura. }\end{array}$ \\
\hline 07 & 2017 & LILACS & $\begin{array}{l}\text { O matriciamento em saúde mental na } \\
\text { perspectiva dos gestores }\end{array}$ & Iglesias \& Avellar. & $\begin{array}{l}\text { Apoio matricial. Saúde mental. } \\
\text { Atenção básica. Gestão. Centro } \\
\text { de atenção psicossocial. }\end{array}$ \\
\hline 08 & 2017 & LILACS & $\begin{array}{l}\text { Apoio matricial na atenção à saúde } \\
\text { mental em uma regional de saúde, } \\
\text { Paraná, Brasil. }\end{array}$ & Garcia et al. & $\begin{array}{l}\text { Saúde mental. Sistemas de apoio } \\
\text { psicossocial. Serviços de saúde } \\
\text { mental. Atenção primária em } \\
\text { saúde. }\end{array}$ \\
\hline 09 & 2018 & SCIELO & $\begin{array}{l}\text { Apoio matricial em saúde mental no } \\
\text { sus de Belo Horizonte: perspectiva } \\
\text { dos trabalhadores }\end{array}$ & Dantas \& Passos. & $\begin{array}{l}\text { Saúde mental. Atenção básica. } \\
\text { Apoio matricial. }\end{array}$ \\
\hline 10 & 2019 & SCIELO & $\begin{array}{l}\text { A contribuição do apoiador matricial } \\
\text { na superação do modelo psiquiátrico } \\
\text { tradicional }\end{array}$ & Silva et al. & $\begin{array}{l}\text { Apoio matricial. Sistema Único } \\
\text { de Saúde. Saúde mental. }\end{array}$ \\
\hline 11 & 2016 & SCIELO & $\begin{array}{l}\text { As contribuições dos psicólogos para } \\
\text { o matriciamento em Saúde Mental }\end{array}$ & Iglesias \& Avellar. & $\begin{array}{l}\text { Atuação do psicólogo. Saúde } \\
\text { Mental. Apoio Matricial. } \\
\text { Integralidade em Saúde. }\end{array}$ \\
\hline 12 & 2019 & SCIELO & $\begin{array}{c}\text { Impasses e desafios para } \\
\text { consolidação e efetividade } \\
\text { do apoio matricial em saúde mental } \\
\text { no Brasil }\end{array}$ & TreicheL et al. & $\begin{array}{l}\text { Apoio matricial. Saúde mental. } \\
\text { Atenção primária à saúde. }\end{array}$ \\
\hline 13 & 2017 & SCIELO & $\begin{array}{c}\text { O "cabo de força” da assistência: } \\
\text { concepção e prática de psicólogos } \\
\text { sobre o Apoio Matricial no Núcleo de } \\
\text { Apoio à Saúde da Família }\end{array}$ & Klein \& D'oliveira. & $\begin{array}{l}\text { Atenção Primária à Saúde. } \\
\text { Psicologia. Saúde Mental. } \\
\begin{array}{l}\text { Equipe de Assistência ao } \\
\text { Paciente. }\end{array}\end{array}$ \\
\hline 14 & 2018 & SCIELO & $\begin{array}{l}\text { Saúde mental na atenção básica: } \\
\text { experiência de matriciamento na área } \\
\text { rural }\end{array}$ & Santos et al. & $\begin{array}{l}\text { Atenção Primária à Saúde. Saúde } \\
\text { Mental. Serviços de Saúde Rural. } \\
\text { Saúde da Família. Relações } \\
\text { Interprofissionais. }\end{array}$ \\
\hline 15 & 2018 & SCIELO & $\begin{array}{l}\text { Tecnologias do cuidado em saúde } \\
\text { mental: práticas e processos da } \\
\text { Atenção Primária }\end{array}$ & Campos et al. & $\begin{array}{l}\text { Assistência Integral à Saúde. } \\
\text { Saúde Mental. Atenção Primária } \\
\text { à } \quad \text { Saúde. Tecnologia. } \\
\text { Enfermagem. }\end{array}$ \\
\hline
\end{tabular}

Fonte: Autores (2021). 
Quanto ao tipo de estudo e metodologias empregadas, constatou-se que todos os artigos selecionados eram estudos do tipo descritivos, sendo, por sua vez, 03 do tipo relatos de experiência, 01 dissertação de mestrado e 11 são artigos de revisão de literatura ou artigos originais, como é possível visualizar no Quadro 2.

Quadro 2: Distribuição de Estudos por tipo, objetivos e resultados principais.

\begin{tabular}{|c|c|c|c|}
\hline $\mathbf{N}^{\mathbf{o}}$ & Tipo de Estudo & Objetivos & Resultados \\
\hline 01 & $\begin{array}{l}\text { Descritivo, } \\
\text { exploratório, } \\
\text { qualitativo. }\end{array}$ & $\begin{array}{l}\text { Identificar o conhecimento dos gestores e } \\
\text { profissionais de saúde de um município do } \\
\text { sertão pernambucano sobre o apoio } \\
\text { matricial em saúde mental. }\end{array}$ & $\begin{array}{l}\text { Evidenciaram-se lacunas de conhecimento sobre } \\
\text { matriciamento e dificuldades para reconhecer as } \\
\text { ações de saúde mental que devem ser } \\
\text { desenvolvidas na Atenção Primária à Saúde. }\end{array}$ \\
\hline 02 & $\begin{array}{l}\text { Descritivo, } \\
\text { prospectivo, } \\
\text { qualitativo. }\end{array}$ & $\begin{array}{l}\text { Conhecer como é realizado o cuidado ao } \\
\text { Portador de Transtorno Mental (PTM) nas } \\
\text { Estratégias de Saúde da Família, } \\
\text { verificando se os profissionais estão } \\
\text { seguros da sua atuação. }\end{array}$ & $\begin{array}{l}\text { Percebe-se que os profissionais se sentem } \\
\text { inseguros para atuarem nessa área de } \\
\text { concentração sendo levantados como } \\
\text { dificultadores a deficiência na teoria científica e a } \\
\text { desarticulação da rede; Matriciamento no cuidado } \\
\text { holístico. }\end{array}$ \\
\hline 03 & $\begin{array}{l}\text { Descritivo, } \\
\text { exploratório. } \\
\text { Método de } \\
\text { Roda. }\end{array}$ & $\begin{array}{l}\text { Identificar os efeitos que os encontros de } \\
\text { matriciamento provocaram no território de } \\
\text { adstringência das equipes da Estratégia } \\
\text { Saúde da Família. }\end{array}$ & $\begin{array}{l}\text { A prática do Apoio Matricial favoreceu a } \\
\text { ampliação das estratégias de cuidados em saúde } \\
\text { mental na Atenção Primária à Saúde; a } \\
\text { corresponsabilidade no cuidado; a escuta } \\
\text { qualificada; o aumento da resolutividade neste } \\
\text { nível de atenção; e a diminuição dos } \\
\text { encaminhamentos sem critérios. }\end{array}$ \\
\hline 04 & $\begin{array}{l}\text { Descritivo, } \\
\text { analítico, } \\
\text { ecológico. }\end{array}$ & $\begin{array}{l}\text { Avaliar a correlação entre indicadores de } \\
\text { estrutura, recursos físicos e trabalhadores, } \\
\text { processo, produção do psicólogo e do } \\
\text { psiquiatra, e a magnitude do matriciamento } \\
\text { realizado. }\end{array}$ & $\begin{array}{l}\text { A produção do psicólogo na atenção básica } \\
\text { aumenta em paralelo a frequência do } \\
\text { matriciamento. A produção aumentada do } \\
\text { psiquiatra, diminui a frequência } \\
\text { matriciamento. }\end{array}$ \\
\hline 05 & $\begin{array}{l}\text { Descritivo, } \\
\text { relato de } \\
\text { experiência. }\end{array}$ & $\begin{array}{l}\text { Implantar o matriciamento em saúde } \\
\text { mental na atenção primária do município } \\
\text { de Toritama-PE. }\end{array}$ & $\begin{array}{l}\text { Foram matriciadas as } 9 \text { Equipes de Saúde da } \\
\text { Família, totalizando } 24 \text { visitas conjuntas, } 38 \\
\text { discussões de casos, } 21 \text { encaminhamentos para o } \\
\text { CAPS, } 46 \text { encaminhamentos para o ambulatório } \\
\text { de saúde mental e } 10 \text { Projetos Terapêuticos } \\
\text { Singular construídos em conjunto. }\end{array}$ \\
\hline 06 & $\begin{array}{l}\text { Descritivo, } \\
\text { exploratório, } \\
\text { revisão de } \\
\text { literatura. }\end{array}$ & $\begin{array}{l}\text { Estabelecer um panorama da produção } \\
\text { científica brasileira sobre o matriciamento } \\
\text { em saúde mental, com foco na articulação } \\
\text { entre a atenção primária e os CAPSs. }\end{array}$ & $\begin{array}{l}\text { Verificou-se que o matriciamento pode contribuir } \\
\text { de diferentes maneiras para a qualificação da } \\
\text { assistência em saúde mental, mas ainda há uma } \\
\text { série de obstáculos. }\end{array}$ \\
\hline 07 & $\begin{array}{l}\text { Descritivo, } \\
\text { Exploratório, } \\
\text { qualitativo. }\end{array}$ & $\begin{array}{l}\text { Analisar as concepções dos gestores sobre } \\
\text { matriciamento em saúde mental, suas } \\
\text { avaliações dos efeitos dessa estratégia e } \\
\text { suas propostas de fortalecimento da } \\
\text { referida prática }\end{array}$ & $\begin{array}{l}\text { Os resultados apontaram três grupos de } \\
\text { entendimento para matriciamento: como } \\
\text { apropriação da temática e do cuidado em saúde } \\
\text { mental pela Atenção Básica, como estratégia de } \\
\text { fiscalização do trabalho da atenção básica e como } \\
\text { dispositivo de formação e orientação para ação } \\
\text { em saúde mental. }\end{array}$ \\
\hline 08 & $\begin{array}{l}\text { Descritivo, } \\
\text { exploratório, } \\
\text { qualitativo. }\end{array}$ & $\begin{array}{l}\text { Analisar os limites e possibilidades do } \\
\text { apoio matricial em saúde mental na } \\
\text { Atenção Básica, segundo a percepção de } \\
\text { gestores e profissionais de saúde em uma } \\
\text { regional de saúde, Paraná, Brasil. }\end{array}$ & $\begin{array}{l}\text { Na primeira categoria: a não realização do } \\
\text { matriciamento por parte do NASF e CAPS e as } \\
\text { contradições entre as políticas de saúde e sua } \\
\text { efetivação. Na segunda, pontua-se a realização e } \\
\text { as potencialidades do matriciamento em saúde } \\
\text { mental. }\end{array}$ \\
\hline
\end{tabular}




\begin{tabular}{|c|c|c|c|}
\hline 09 & $\begin{array}{c}\text { Descritivo, } \\
\text { exploratório, } \\
\text { qualitativo. }\end{array}$ & $\begin{array}{l}\text { Analisar a prática do apoio matricial em } \\
\text { saúde mental com base no estudo de caso } \\
\text { da experiência da cidade de Belo } \\
\text { Horizonte, Minas Gerais, ocorrida entre os } \\
\text { anos de 2013-2014. }\end{array}$ & $\begin{array}{l}\text { Percebeu-se que o apoio matricial tem } \\
\text { transformado a relação de trabalho nas unidades } \\
\text { básicas de saúde no sentido da } \\
\text { corresponsabilização e do trabalho integrado entre } \\
\text { as equipes. }\end{array}$ \\
\hline 10 & $\begin{array}{l}\text { Descritivo, } \\
\text { relato de } \\
\text { experiência. }\end{array}$ & $\begin{array}{l}\text { Relatar o processo de trabalho do apoiador } \\
\text { matricial para o cuidado em saúde mental } \\
\text { na atenção básica, na perspectiva da } \\
\text { superação do modelo psiquiátrico } \\
\text { tradicional. }\end{array}$ & $\begin{array}{l}\text { A experiência evidenciou que as atividades de } \\
\text { matriciamento potencializaram a atuação das } \\
\text { equipes da Atenção Básica para as demandas de } \\
\text { saúde mental no território. }\end{array}$ \\
\hline 11 & $\begin{array}{c}\text { Descritivo, } \\
\text { exploratório, } \\
\text { qualitativo. }\end{array}$ & $\begin{array}{l}\text { Investigar as concepções do psicólogo da } \\
\text { atenção básica sobre o apoio matricial em } \\
\text { saúde mental e as consequentes } \\
\text { repercussões de tais entendimentos para } \\
\text { sua prática neste nível de atenção. }\end{array}$ & $\begin{array}{l}\text { Alguns psicólogos apresentaram dificuldades em } \\
\text { afirmar outra prática que não aquela voltada ao } \\
\text { atendimento ambulatorial individual, mas } \\
\text { também outros conseguiram estabelecer parcerias } \\
\text { diversas para o cuidado em saúde, a partir da } \\
\text { concepção de que este trabalho requer } \\
\text { compartilhamento de saberes, para uma atuação } \\
\text { voltada à integralidade. }\end{array}$ \\
\hline 12 & $\begin{array}{c}\text { Descritivo, } \\
\text { exploratório, } \\
\text { revisão } \\
\text { integrativa. }\end{array}$ & $\begin{array}{l}\text { Revisar a bibliografia nacional dos últimos } \\
\text { dez anos a fim de identificar os impasses e } \\
\text { desafios vivenciados no apoio matricial em } \\
\text { saúde mental na Atenção Primária, } \\
\text { classificando-os a partir de uma } \\
\text { reconstrução teórico-conceitual e fazendo } \\
\text { uma articulação destes com os desafios } \\
\text { pontuados em congêneres internacionais } \\
\text { do apoio matricial. }\end{array}$ & $\begin{array}{l}\text { Destacou-se a necessidade de delineamentos } \\
\text { claros para prática de matriciamento; } \\
\text { investimento maciço em formação e capacitação } \\
\text { dos profissionais; e criação de espaços } \\
\text { institucionalizados com encontros sistemáticos } \\
\text { dos profissionais para discussão dos casos e } \\
\text { avaliação conjunta do andamento das atividades. }\end{array}$ \\
\hline 13 & $\begin{array}{l}\text { Descritivo, } \\
\text { qualitativo. }\end{array}$ & $\begin{array}{l}\text { Analisar a concepção e a prática do } \\
\text { matriciamento realizadas por psicólogos } \\
\text { que trabalham no Núcleo de Apoio à } \\
\text { Saúde da Família (NASF) no Município de } \\
\text { São Paulo, Brasil. }\end{array}$ & $\begin{array}{l}\text { A troca de saberes e a capacitação apareceram } \\
\text { como principais significados da concepção de } \\
\text { matriciamento. A prática revelou um contexto } \\
\text { complexo com uma organização de trabalho } \\
\text { bastante heterogênea com falta de articulação } \\
\text { entre gerências e tensões na execução do trabalho } \\
\text { compartilhado. }\end{array}$ \\
\hline 14 & $\begin{array}{l}\text { Descritivo, } \\
\text { relato de } \\
\text { experiência. }\end{array}$ & $\begin{array}{l}\text { Relatar o desenvolvimento de ações de } \\
\text { Saúde Mental compartilhadas entre a } \\
\text { Estratégia Saúde da Família alocada em } \\
\text { uma área rural e o Núcleo de Apoio } \\
\text { Matricial, evidenciando as interlocuções } \\
\text { decorrentes dessa configuração singular. }\end{array}$ & $\begin{array}{l}\text { Foram identificadas as necessidades de saúde: } \\
\text { consumo de psicofármacos, escassez de } \\
\text { atividades para o cuidado coletivo e dificuldade } \\
\text { de acesso ao serviço. Conforme as atitudes } \\
\text { educacionais foram implementadas, a ampliação } \\
\text { das ações e o envolvimento dos atores com a } \\
\text { intersetorialidade ficaram evidentes. }\end{array}$ \\
\hline 15 & $\begin{array}{l}\text { Descritivo, } \\
\text { qualitativo }\end{array}$ & $\begin{array}{l}\text { Analisar as tecnologias do cuidado em } \\
\text { saúde mental utilizadas nas práticas e } \\
\text { processos constituintes da Atenção } \\
\text { Primária à Saúde a partir dos discursos de } \\
\text { enfermeiros da Estratégia Saúde da } \\
\text { Família. }\end{array}$ & $\begin{array}{l}\text { O estudo apontou o acolhimento e o } \\
\text { matriciamento como as principais tecnologias de } \\
\text { cuidado exercidas na interface da Atenção } \\
\text { Primária à Saúde com a Saúde Mental. } \\
\text { Entretanto, evidenciou-se a necessidade de ações } \\
\text { fortalecedoras para o matriciamento, para a } \\
\text { capacitação no sentido de melhorar a autonomia } \\
\text { dos profissionais diante dessa demanda. }\end{array}$ \\
\hline
\end{tabular}

Fonte: Autores (2021).

Os resultados dos estudos 01, 02, 08, 11 e 14 encontrados evidenciaram em sua maior parte as principais dificuldades encontradas para a implantação ou concretização do modelo matricial em saúde mental. Dentre estes fatores, observou-se a 
falta de conhecimento dos profissionais quanto este modelo de atenção à saúde, as dificuldades em lidar com pacientes com transtornos mentais, que desarticulam a rede de atenção primária.

As demais publicações referem, experiências em locais nos quais os modelos de matriciamento foram adotados, e ainda, as principais contribuições para as redes de atenção à saúde.

\section{Discussão}

A partir da análise dos artigos, foram identificadas duas categorias. O critério para a escolha destas foi semântico, isto é, as ideias ou contextos que apresentaram semelhança foram agrupados, resultando em duas 1) benefícios e resultados do modelo matricial; 2) fatores que interferem na implantação do matriciamento.

\section{Benefícios e resultados do modelo matricial em saúde mental}

O matriciamento ou apoio matricial é uma construção de uma proposta de intervenção terapêutico-pedagógica, realizada de forma compartilhada, que envolve duas ou mais equipes. Visa realizar assistência especializada, promovendo vínculos profissionais e instituindo projetos coletivos terapêuticos, junto aos usuários e à comunidade (Vasconcelos \& Barbosa, 2019).

A proposta de apoio matricial brasileira possui o compromisso com uma construção de relacionamentos democráticos a partir da cogestão e construção do cuidado de forma compartilhada. Prevê-se que haja comunicação e diálogo entre os profissionais de saúde e os usuários, para que estes sejam protagonistas na elaboração de seu plano de cuidados (Treichel et al, 2019).

Campos, Bezerra e Jorge (2018), verificaram em seu estudo, realizado em Fortaleza-CE, que enfermeiros citam o matriciamento em saúde como uma tecnologia de cuidado, e que por meio desta, ações da atenção primária à saúde passam a ser reflexivas, inventivas e embasadas nas necessidades do usuário.

A dinâmica e interação nos encontros propicia a troca de experiências entre profissionais, e favorece, através dos espaços de educação permanente a exposição de dificuldades à aproximação com pacientes de transtornos psíquicos em seu território (Silva et al, 2019).

Belotti e Lavrador (2016), constataram em seus estudos que as ações de saúde mental, realizadas pelas equipes de uma Estratégia de Saúde da Família, antes da implantação do matriciamento, eram restritas a encaminhamentos a serviços de saúde mental, sem acolhimento dos pacientes com transtornos mentais.

Silva et al, (2019), relatam que a inserção apoio matricial na organização do município de João Pessoa-PB no suporte às equipes das ESF, aperfeiçoou a comunicação entre os serviços especializados e a atenção básica, promovendo encontros de experiências, proporcionando, por sua vez, uma atenção mais integral, de forma menos fragmentada.

Gonçalves e Peres (2018) mencionam como os principais fatores que favorecem o matriciamento: a visão ampliada do processo saúde-doença, equipamentos intersetoriais no território, a realização de intervenções coletivas e pactuações de classificação de risco.

\section{Fatores que interferem na implantação do matriciamento em saúde mental}

A fragmentação da rede de saúde com baixa integração, ou ainda, a burocratização dos serviços, torna-se uma dificuldade para os pressupostos da proposta matricial, o que perpetua o número de encaminhamentos, havendo, ainda, a descontinuidade das ações entre os níveis de atenção à saúde (Treichel et al, 2019).

Garcia et al. (2017) observou, através de falas profissionais, que os serviços especializados que prestariam o apoio matricial não possuem ciência de suas funções inseridas na rede. Evidenciou-se também nos estudos de Silva et al. (2017) que 
as equipes das ESF não conhecem ou se aproximam dos serviços do CAPS e ambulatório de psiquiatria, não havendo clareza no entendimento das funções desempenhadas por estas instituições.

Associado a isso, sabe-se que o Centro de Atenção Psicossocial (CAPS) não possui a capacidade de atender toda a demanda em saúde mental. Sendo assim, a ausência de articulações entre este e a atenção básica, centraliza os serviços, fazendo com que os serviços especializados sejam menos inseridos na comunidade e no território (Garcia et al, 2017).

Sendo Almeida et al. (2020) é comum observar o sentimento de medo presente em profissionais ao lidar com pacientes de saúde mental, visto que há falta de conhecimento ou especializações na área, ou ainda, estes sentem-se ameaçados mediante a reação dos usuários. Não obstante, há ainda, a estigmatização e exclusão social (Iglesias \& Avellar, 2017). Tais fatores mostram-se relevantes ao enfraquecer a política e modelo atuais.

Em um estudo realizado em área rural, realizado por Santos et al. (2018) verificou-se que há uma tendência da valorização do profissional da medicina e da medicalização, o que possui baixa eficácia se não associado às intervenções psicossociais. Além disso, outros profissionais da saúde são concebidos como atuantes secundários no cuidado, tornando, assim, a assistência desconectada e fragmentada.

Iglesias e Avellar (2016) acreditam que a efetivação do apoio matricial está estreitamente ligada ao entendimento dos profissionais acerca do matriciamento, assim como das demais profissões que compões a atenção básica, além da psicologia.

\section{Conclusão}

Através desta revisão integrativa de literatura, buscou-se encontrar na literatura as principais referências relacionadas ao matriciamento em saúde mental na atenção primária no Brasil. Referente à metodologia, todos os estudos possuíam caráter descritivo.

Quanto aos resultados aqui explicitados, notou-se a maior parte dos estudos referiram os benefícios da aplicação desta estratégia, como por exemplo a integração do processo saúde doença, valorizando a equipe multiprofissional, de forma que esta dialogue com o portador de transtorno mental e sua família, tornando-o protagonista em seu plano de cuidado.

Apesar de ser um plano estratégico que mostra resultados, o apoio matricial encontra diversas dificuldades em sua implantação, sejam estas estruturais, por falta de capacitação técnica dos profissionais atuantes na rede de saúde ao lidar com pacientes com estas condições, seja pela centralização do cuidado na medicalização das patologias.

Dessa forma, faz-se necessário que os profissionais atuantes na rede psicossocial, conheçam as suas funções desempenhadas como matriciadores no processo de saúde mental, contribuindo, por sua vez, com a descentralização dos serviços ao integrar a atenção básica aos serviços especializados. Assim, com o matriciamento implantado, o usuário passa a ter um cuidado holístico e humanizado por toda a equipe da rede de atenção psicossocial.

\section{Referências}

Almeida, D. R., Soares, J. N. C., Dias, M. G., Rocha, F. C., Andrade Neto, G. R. D., \& Andrade, D. L. B. (2020). O cuidado aos portadores de sofrimento mental na atenção primária: uma prática interdisciplinar e multiprofissional. Revista Online de Pesquisa, 454-459, 2 (12).

Almeida, J. M. C. D. (2019). Política de saúde mental no Brasil: o que está em jogo nas mudanças em curso. Cadernos de Saúde Pública, 35 , e00129519.

Almeida, L. P. V. G. D., \& Ferraz, C. A. (2008). Políticas de formação de recursos humanos em saúde e enfermagem. Revista Brasileira de Enfermagem, 61(1), 31-35.

Belotti, M., \& Lavrador, M. C. C. (2016). A prática do apoio matricial e os seus efeitos na Atenção Primária à Saúde/The practice of matrix support and its effects on primary health care. Cadernos Brasileiros de Terapia Ocupacional, 24(2), 373-378.

Campos, D. B., Bezerra, I. C., \& Jorge, M. S. B. (2018). Tecnologias do cuidado em saúde mental: práticas e processos da Atenção Primária. Revista Brasileira de Enfermagem, 71(5), 2101-2108. 
Campos, F. E., Ferreira, J. R., Feuerwerker, L., de Sena, R. R., Campos, J. J. B., Cordeiro, H., \& Cordoni Jr, L. (2001). Caminhos para aproximar a formação de profissionais de saúde das necessidades da atenção básica. Revista Brasileira de Educação Médica, 25(2), 53-59.

Carvalho, Y. M., \& Ceccim, R. B. (2006). Formação e educação em saúde: aprendizados com a saúde coletiva. Tratado de saúde coletiva, (2a ed.) 149-82, $137-170$.

Dantas, N. F., \& Passos, I. C. F. (2018). El apoyo de matriz en salud mental en el sistema único de salud de belo horizonte: perspectiva de los trabajadores. Trabalho, Educação e Saúde, 16(1), 201-220.

Faro, A., Bahiano, M. D. A., Nakano, T. D. C., Reis, C., Silva, B. F. P. D., \& Vitti, L. S. (2020). COVID-19 e saúde mental: a emergência do cuidado. Estudos de Psicologia (Campinas), 37.

Garcia, G. D. V., da Silva, I. F., Cavalcante, M., da Rocha Cervo, M., Zambenedetti, G., \& Zanoti-Jeronymo, D. V. (2017). Apoio matricial na atenção à saúde mental em uma regional de saúde, Paraná, Brasil. Saúde e Pesquisa, 10(3), 423-432.

Godoi, L. P. D. S. (2020). Apoio matricial como ferramenta da articulação entre Atenção Básica e CAPS. Doctoral dissertation, Universidade de São Paulo.

Iglesias, A., \& Avellar, L. Z. (2016). As contribuições dos psicólogos para o matriciamento em saúde mental. Psicologia: Ciência e Profissão, 36(2), 364-379. Klein, A. P., \& d'Oliveira, A. F. P. L. (2017). O" cabo de força" da assistência: concepção e prática de psicólogos sobre o Apoio Matricial no Núcleo de Apoio à Saúde da Família. Cadernos de Saúde Pública, 33, e00158815.

Macedo, J. P., Abreu, M. M. D., Fontenele, M. G., \& Dimenstein, M. (2017). A regionalização da saúde mental e os novos desafios da Reforma Psiquiátrica brasileira. Saúde e Sociedade, 26, 155-170.

Marin, M. J. S., Lima, E. F. G., Paviotti, A. B., Matsuyama, D. T., Silva, L. K. D. D., Gonzalez, C., \& Ilias, M. (2010). Aspectos das fortalezas e fragilidades no uso das metodologias ativas de aprendizagem. Revista brasileira de educação médica, 34(1), 13-20.

Ministério da Saúde (BR) (1997). Saúde da Família: uma estratégia para a reorientação do modelo assistencial. Secretaria de Assistência à Saúde. Coordenação de Saúde da Comunidade. Brazilian-DF, p. 36, 1997. http://bvsms.saude.gov.br/bvs/publicacoes/cd09_16.pdf.

Ministério da Saúde (BR) (2011). Guia prático de matriciamento em saúde mental. Centro de Estudo e Pesquisa em Saúde Coletiva. Brazilian-DF. http://bvsms.saude.gov.br/bvs/publicacoes/guia_pratico_matriciamento_saudemental.pdf.

Santos, L. C. D., Domingos, T. D. S., Braga, E. M., \& Spiri, W. C. (2020). Saúde mental na atenção básica: experiência de matriciamento na área rural. Revista Brasileira de Enfermagem, 73(10), 1-5.

Silva, F. C. (2017). Implantação do matriciamento em saúde mental na atenção básica do município de Toritama-PE. Escola do Governo de Saúde de Pernanbuco, $1-10$

Souza, M. D., Silva, M. D., \& Carvalho, R. D. (2010). Integrative review: what is it? How to do it? Einstein, 8 (1), $102-106$.

Treichel, C. A. D. S., Campos, R. T. O., \& Campos, G. W. D. S. (2019). Impasses e desafios para consolidação e efetividade do apoio matricial em saúde mental no Brasil. Interface-Comunicação, Saúde, Educação, 23, e180617.

Vasconcelos, M. S., \& Barbosa, V. F. B. (2019). Conhecimento de gestores e profissionais da rede de atenção psicossocial sobre matriciamento em saúde mental. Cienc Cuid Saude, 18(4), e43922. 